\title{
Theoretical Studies on the $\alpha$-decay Half-Lives of Even-Even Lv Isotopes
}

\author{
Dashty T. Akrawy ${ }^{1,2}$ \\ ${ }^{1}$ Akre Computer Institute, Ministry of Education, Aqrah, Kurdistan, Iraq \\ ${ }^{2}$ Becquereal Institute for Radiation Research and Measurements, Erbil, Kurdistan, Iraq
}

Email address:

akrawy85@gmail.com

\section{To cite this article:}

Dashty T. Akrawy. Theoretical Studies on the $\alpha$-decay Half-Lives of Even-Even Lv Isotopes. International Journal of Energy and Power Engineering. Vol. 6, No. 1, 2017, pp. 1-5. doi: 10.11648/j.ijepe.20170601.11

Received: February 19, 2017; Accepted: March 13, 2017; Published: March 23, 2017

\begin{abstract}
The $\alpha$-decay half-lives for even-even $\mathrm{Lv}$ isotopes within the range $290 \leq \mathrm{A} \geq 314$ which have $\mathrm{Z}=116$, have been evaluated using Gamow-like model (GLM) which is based on Gamow theory, the different semi-empirical formula for $\alpha$-decay half-lives also was used. The half-lives were evaluated using theoretical $Q$-value. The computed $\alpha$-decay half-lives were compared with CPPM, and also with empirical formula, the result was achieved in a good agreement.
\end{abstract}

Keywords: $\alpha$-decay; Gamow-Like Model; Semi-empirical Formula

\section{Introduction}

The $\alpha$-decay half-lives is one of the most important decay modes of nuclei which was described in 1928 [1] in base of quantum tunneling, who assumed the $\alpha$-particle was due to the quantum tunneling phenomenon of a charged $\alpha$-particle through the Coulomb barrier. Violet and Seaborg was predictive a simple formula which is based on Gamow model [2]. Poenaru et. al was predicted theoretical model of cluster radioactivity [3]. The cluster radioactivity phenomenon may be described in a similar way as the alpha decay using Gamow-like theories [2, 4] because of the large mass difference of cluster and daughter nucleons. A simple formula for $\alpha$-decay half-lives was derived using WentzelKramers-Brillouin (WKB) approximation theory by A. Zdeb et al [2] for penetration of the Coulomb barrier with a square well for the nuclear part. GLM depended only one adjustable parameter, the radius constant, which is possible to reproduce with a good accuracy all existing data for decays of eveneven nuclei. To determine a $\alpha$-decay half-lives there are more than model that was used as shown in the literature such as Royer, he generalized the liquid drop model to describe $\alpha$ decay half-lives [5], and that formula was used to evaluate the $\alpha$-decay half-lives for heavy and superheavy nuclei [6]. Ibrahim et. al determined the $\alpha$-decay preformation probability of superheavy nuclei within the binary cluster model by using an interaction generated from amalgamation of a phenomenological and microscopic interactions [7]. Yao et. al was used different proximity potentials to evaluate $\alpha$ decay half-lives, which was include Coulomb proximity potential model and Coulomb proximity potential model for different deformation nuclei [8]. Sun et. al studied $\alpha$-decay half-live for even-even nuclei by using two potential approaches with a quasistationary state approximation [9]. Javadimanesh et. al also studied $\alpha$-decay half-lives by considering a potential model with Yukawa proximity potential from ground state to ground state [10]. The aim of this work is to determine the $\alpha$-decay half-lives of even-even Lv isotopes within Gamow-Like Model (GLM) and to compared with another theoretical model such as CPPM [11], Royer formula [12], Universal Decay Low (UDL) [13], Viola-Seaborg formula (VSS) [14], Semi-empirical formula for Poenaru et. Al. (SemFIS) [15], and finally with Denisov \& Khudenko formula (DEKH) [16].

\section{Gamow-Like Model for $\alpha$-decay Half-Lives}

A simple phenomenological model for evaluation of $\alpha$ decay half-lives which is based on the Gamow theory was developed by A. Zdeb et. al [2], this model is contain one adjusted parameter which is Coulomb potential. 
The probability of tunneling $\alpha$-particle through the potential barrier was given within (WKB) approximation theory by the following integral $[2,17]$

$$
\boldsymbol{P}=\exp \left[-\frac{2}{\hbar} \int_{\boldsymbol{R}}^{b} \sqrt{2 \mu(\boldsymbol{V}(\boldsymbol{r})-\boldsymbol{Q})} d \boldsymbol{r}\right]
$$

Where $Q$, is kinetic energy of the emitted particle, $\mu$ is the reduced mass. The spherical square well radius $R$, which is equal to sum of the radii of parent and daughter nuclei,

$$
\boldsymbol{R}=\boldsymbol{r}_{0}\left(\boldsymbol{A}_{1}^{1 / 3}+\boldsymbol{A}_{2}^{1 / 3}\right)
$$

$\boldsymbol{A}_{1}$ and $\boldsymbol{A}_{2}$ are atomic number of parent and daughter nuclei. $\boldsymbol{r}_{0}$ has been taken from Ref. [5]

The exit point from the barrier $\mathrm{b}$ corresponding to point, where the Coulomb potential is equal to the kinetic energy $Q$ :

$$
b=\frac{Z_{1} Z_{2} e^{2}}{Q}
$$

where $Z_{1}$ and $Z_{2}$ are the atomic number of parent and daughter nuclei respectively, $e^{2}$ is the electron charge given by $e^{2}=1.43998 \mathrm{MeV} . \mathrm{fm}$ [18]. The $Q$ value was taken from theoretical value [11].

The potential energy $V(r)$ is given by

$$
\boldsymbol{V}(\boldsymbol{r})= \begin{cases}-\boldsymbol{V}_{0} & 0 \leq \boldsymbol{r} \leq \boldsymbol{R} \\ \frac{\boldsymbol{Z}_{1} \boldsymbol{Z}_{2} \boldsymbol{e}^{2}}{\boldsymbol{r}} & \boldsymbol{r}>\boldsymbol{R}\end{cases}
$$

Where the depth of the potential well $V_{o}$ is one of the model parameters which is equal to $V_{0}=25 A_{1}$ [19]. The $\alpha$-decay half-life can be calculated is given by the following [20]

$$
\boldsymbol{T}_{1 / 2}=\frac{\ln 2}{\lambda}
$$

the decay constant is simply defined as $\lambda=\boldsymbol{v P}, v$ is the assault frequency which is equal to $10^{20} \mathrm{~s}^{-1}$ [21], the penetration probability $P$ was calculated by using integral of Eq. (1).

\section{Empirical Formulas for $\alpha$-decay Half-Lives}

Some empirical formulas of $\alpha$-decay half-lives for $\mathrm{Pb}$ isotopes were evaluated in this study for comparison of our calculation with GLM model by using the following empirical formulae.

\subsection{Royer Empirical Formula (Royer)}

The $\alpha$-decay half-lives have been studied by using Royer formula [12, 22-23], Royer developed formula by fitting procedure using 373 nuclei transition from ground state to ground state which is given as

$$
\log _{10}\left[\boldsymbol{T}_{1 / 2}(\boldsymbol{s})\right]=\boldsymbol{a}-\boldsymbol{b} \boldsymbol{A}^{1 / 6} \sqrt{\boldsymbol{Z}}+\frac{\boldsymbol{c} \boldsymbol{Z}}{\sqrt{\boldsymbol{Q}}}
$$

where $\mathrm{A}, \mathrm{Z}$ and $\mathrm{Q}$ represented mass number, atomic number and energy released during the reaction. The Eq.(6) can be assumed for set even-even nuclei, where the coefficients $a, b$, and $c$ is obtained by least square fitting methods which is $25.31,-1.169$ and 1.5864 respectively for even-even nuclei.

\subsection{Universal Decay Law Formula (UDL)}

From the microscopic mechanism of the radioactive decay charge particle emission, the universal decay law for alpha decay and cluster was predicted by Qi et al [13], It connected the half-lives of monopole radioactive decays with the kinetic energy $(Q)$ values of the outgoing particles as well as the atomic number and mass number of the nuclei implicated in the decay $[24,25]$. The relation is found to be as

$$
\begin{aligned}
\log _{10}\left[T_{1 / 2}(s)\right] & =a Z_{c} Z_{d} \sqrt{\frac{A}{Q_{c}}}+b \sqrt{A Z_{c} Z_{d}\left(A_{d}^{1 / 3}+A_{c}^{1 / 3}\right)}+c \\
& =a \chi^{\prime}+b \rho^{\prime}+c
\end{aligned}
$$

where $A=\sqrt{\frac{\boldsymbol{A}_{d} \boldsymbol{A}_{c}}{\left(\boldsymbol{A}_{d}+\boldsymbol{A}_{c}\right)}}$, and the constant $a=0.3949$, $b=-0.3693$ and $c=-23.7615$ are constants which are used was determined by fitting to the experimental data of $\alpha$-decay and cluster [26]. $\boldsymbol{b} \rho^{\prime}+\boldsymbol{c}$ is the effects term that include the cluster in the parent nucleus.

\subsection{The Viola-Seaborg Semi-empirical Formula (VSS)}

From the Geiger-Nuttall law, the VSS formula was determined by Sobiczewski et al. [27], is given as

$$
\log _{10}\left[\boldsymbol{T}_{1 / 2}(s)\right]=\frac{(\boldsymbol{a} \boldsymbol{Z}+\boldsymbol{b})}{\sqrt{\boldsymbol{Q}}}+\boldsymbol{c} \boldsymbol{Z}+\boldsymbol{d}+\boldsymbol{h}_{\mathrm{log}}
$$

Here $Z$ is the parent atomic number, $Q$ is the energy released during the reaction in unit $\mathrm{MeV}$, half-life in seconds, the coefficients $a, b, c$, and $d$ are adaptable parameters and the parameter $h_{\text {log }}$ evidence the hindrance factor related with odd proton and odd neutron numbers [28], as given by Viola et al. [29]. More recent value was determined by Tiekuang Dong et. Al [14], new data for even-even nuclei is taken into, have been used to determine the constant which is given as $a$ $=1.64062, b=-8.54399, c=-0.19430, d=-33.9054$, and $h_{l o g}$ $=0$ for even even nuclei.

\subsection{Semi-empirical Formula for Poenaru et al. (SemFIS)}

Poenaru et al. modified semi-empirical formula for $\alpha$ decay half-life which is based on fission theory for $\alpha$-decay yield which is given as $[15,30]$

$$
\log _{10}\left[\boldsymbol{T}_{1 / 2}(s)\right]=0.43429 \boldsymbol{K}_{s} \chi-20.446
$$


where

$$
\boldsymbol{K}_{s}=2.52956 \boldsymbol{Z}_{d}\left[\frac{\boldsymbol{A}_{d}}{\boldsymbol{A \boldsymbol { Q } _ { \alpha }}}\right]^{1 / 2}[\arccos \sqrt{\boldsymbol{r}}-\sqrt{\boldsymbol{r}(1-\boldsymbol{r})}]
$$

and

$$
\boldsymbol{r}=0.423 \boldsymbol{Q}_{\alpha} \frac{\left(1.5874+\boldsymbol{A}_{d}^{1 / 3}\right)}{\boldsymbol{Z}_{d}}
$$

and the numerical coefficient $\chi$, close to the unity, is a second order polynomial

$$
\chi=B_{1}+B_{2} y+B_{3} z+B_{4} y^{2}+B_{5} y z+B_{6} z^{2}
$$

The reduced variable $y$ and $z$, expressed the distance from the closest magic-plus-one neutron and proton numbers $N_{i}$ and $Z_{i}$ is given as

$$
\begin{array}{ll}
z=\frac{\left(N-N_{i}\right)}{\left(N_{i+1}-N_{i}\right)}, & N_{i}<N \leq N_{i+1} \\
y=\frac{\left(Z-Z_{i}\right)}{\left(Z_{i+1}-Z_{i}\right)}, & Z_{i}<Z \leq Z_{i+1}
\end{array}
$$

with $N_{i}=\ldots, 51,83,127,185,229, \ldots$, and $Z_{i}=\ldots, 29,51$, $83,127, \ldots$; Bi have been obtained by using a high quality selected data set of $\alpha$-decay which is equal to $B_{1}=0.993119$, $B_{2}=-0.004670, B_{3}=0.01701, B_{4}=10.04503, B_{5}=0.018102$, and $B_{6}=-0.025097$.

\subsection{Denisov \& Khudenko Formula (DEKH)}

From the Guy Royer empirical formula [23], Denisov \& Khudenko $[16,31]$ developed empirical formula for $\alpha$-decay half-life between ground state to ground state $\alpha$-transition of parent and daughter nuclei, the formula is evaluated of $\alpha$ decay half-lives for even-even, even-odd, odd-even and oddodd nuclei:

$$
\log _{10}\left[\boldsymbol{T}_{1 / 2}(s)\right]=-\boldsymbol{a}-\frac{\boldsymbol{b} \boldsymbol{A}^{1 / 6} \sqrt{\boldsymbol{Z}}}{\mu}+\frac{\boldsymbol{c} \boldsymbol{Z}}{\sqrt{\boldsymbol{Q}_{\alpha}}}+\frac{\boldsymbol{d} \sqrt{\ell(\ell+1)}}{\boldsymbol{Q} \boldsymbol{A}^{-1 / 6}}-e\left((-1)^{\ell}-1\right)
$$

Here $\mathrm{A}$ and $\mathrm{Z}$ are the mass number and atomic number of parent nucleus, respectively, $\ell$ is the orbital moment of emitted $\alpha$ particle, and $\mu=(\boldsymbol{A} /(\boldsymbol{A}-4))^{1 / 6} \cdot Q$ is the reaction energy value. The $\alpha$-particle emission from nuclei obeys the spin-parity selection rule [32, 33]

$$
\ell_{\min }= \begin{cases}\Delta j & \text { for even } \Delta \boldsymbol{j} \text { and } \pi_{p}=\pi_{d} \\ \Delta j+1 & \text { for odd } \Delta \boldsymbol{j} \text { and } \pi_{p}=\pi_{d} \\ \Delta \boldsymbol{j} & \text { for odd } \Delta \boldsymbol{j} \text { and } \pi_{p} \neq \pi_{d} \\ \Delta \boldsymbol{j}+1 & \text { for even } \Delta \boldsymbol{j} \text { and } \pi_{p} \neq \pi_{d}\end{cases}
$$

where $\Delta \boldsymbol{j}=\left|\boldsymbol{j}_{p}-\boldsymbol{j}_{d}\right|, \boldsymbol{j}_{p}, \boldsymbol{\pi}_{p}, \boldsymbol{j}_{\boldsymbol{d}}, \boldsymbol{\pi}_{\boldsymbol{d}}$ are spin and parity values of the parent and daughter nuclei, respectively, and $\ell=\ell_{\min }$, the $a, b, c, d$, and $e$ are free parameters have been evaluated by Denisov et al. [31] which are 26.1721, 1.1549, 1.6088, 0 , and 0 respectively.

\section{Results and Discussions}

The theoretical $\alpha$-decay half-lives of even-even Lv isotopes, which have atomic number $Z=118$ within the range $\mathrm{A}=178-220$ have been studied by using Gamow-Like Model. The $Q$-value was taken in Ref. [11] which is evaluated theoretically are listed in Table 1 . The evaluation of $\alpha$-decay half-lives within GLM are given in Table 1.

The semi-empirical formulae was also been done using the

\begin{tabular}{|c|c|c|c|c|c|c|c|c|}
\hline \multirow{2}{*}{$\mathbf{A}$} & \multirow{2}{*}{$\mathbf{Q}$} & \multicolumn{7}{|c|}{$\log _{10}\left(T_{1 / 2}\right)(s)$} \\
\hline & & GLM & СРРМ & Royer & UDL & VSS & SemFIS & DEKH \\
\hline 290 & 11.054 & -2.024 & -1.279 & -1.677 & -0.976 & -1.360 & -1.349 & -1.275 \\
\hline 292 & 10.834 & -1.498 & -0.710 & -1.146 & -0.452 & -0.790 & -0.765 & -0.736 \\
\hline 294 & 10.224 & 0.120 & 1.024 & 0.489 & 1.145 & 0.886 & 0.935 & 0.922 \\
\hline 296 & 10.564 & -0.850 & -0.009 & -0.498 & 0.193 & -0.066 & 0.006 & -0.078 \\
\hline 298 & 10.324 & -0.224 & 0.664 & 0.131 & 0.812 & 0.601 & 0.707 & 0.560 \\
\hline 300 & 10.194 & 0.111 & 1.024 & 0.465 & 1.144 & 0.972 & 1.115 & 0.899 \\
\hline 302 & 11.784 & -3.930 & -3.336 & -3.670 & -2.870 & -3.135 & -2.983 & -3.294 \\
\hline 304 & 10.944 & -1.963 & -1.190 & -1.652 & -0.901 & -1.077 & -1.943 & -1.247 \\
\hline 306 & 10.184 & 0.047 & 0.970 & 0.385 & 1.088 & 1.001 & 0.035 & 0.820 \\
\hline 308 & 9.424 & 2.323 & 3.385 & 2.669 & 3.316 & 3.325 & 2.280 & 3.136 \\
\hline 310 & 8.474 & 5.639 & 6.854 & 5.960 & 6.524 & 6.659 & 5.552 & 6.475 \\
\hline 312 & 8.214 & 6.631 & 7.887 & 6.935 & 7.479 & 7.670 & 6.555 & 7.464 \\
\hline 314 & 7.984 & 7.549 & 8.840 & 7.833 & 8.361 & 8.606 & 7.493 & 8.376 \\
\hline
\end{tabular}
analytical Royer formula (Royer), the universal decay law (UDL), the Viola-Seaborg formula (VSS), the Semiempirical formula for Poenaru et. Al. (SemFIS), and finally analytical Denisov \& Khudenko formula (DEKH) have also been evaluated and presented in Table 1.

Table 1. The comparison of evaluated logarithm $\alpha$-decay half-lives of even-even Lv isotopes.

The comparison between GLM, CPPM, and some empirical formulae such as Royer, UDL, VSS, SemFIS and DEKH have been done and presented in Table 1 , it can be found that the results that the $\alpha$-decay half-lives evaluated using GLM are in good agreement with other theoretical model and empirical formulae. 
The plot for logarithm $\left(T_{1 / 2}\right)$ versus the neutron number of the parent nuclei of the $\alpha$-emissions from even-even Lv isotopes are shown in Figure 1.

From Figure 1 it's noted that, the maximum value of logarithm $\left(T_{1 / 2}\right)$ half-life is for the decay of the parent nuclei ${ }^{314} \mathrm{Lv}$.

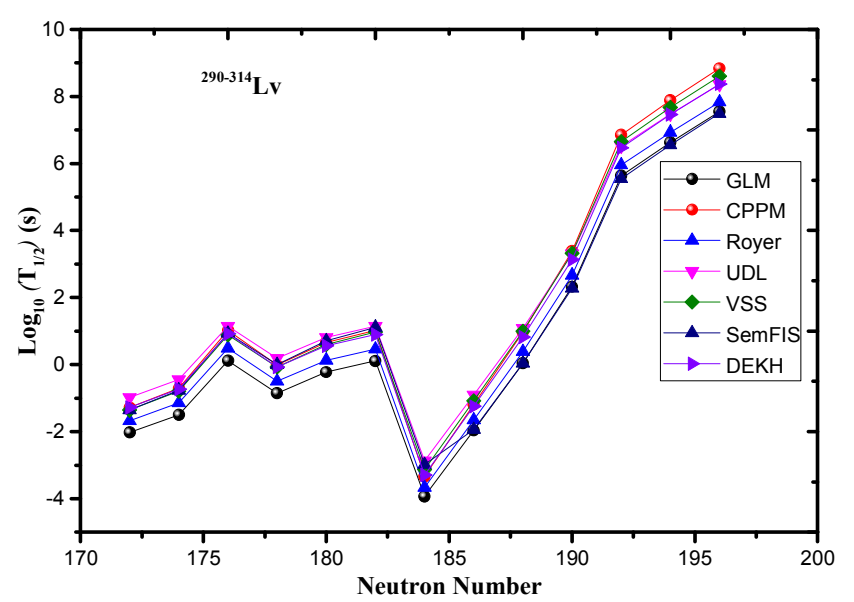

Figure 1. Comparison of the evaluated $\alpha$-decay half-lives of even-even $L v$ isotopes.

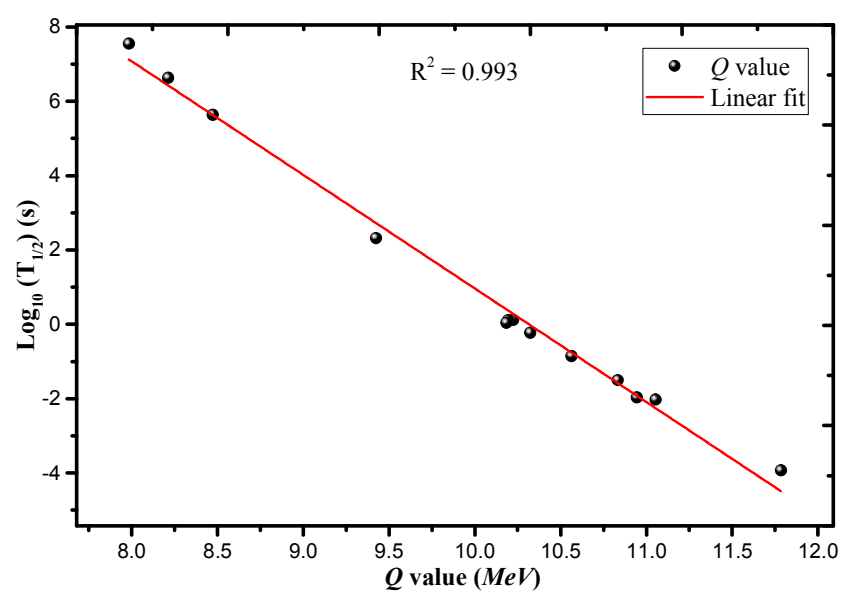

Figure 2. Correlation between $Q$ value versus Logarithm $\left(T_{1 / 2}\right) \alpha$-decay halflives of Lv isotopes.

The minimum value of logarithm $\left(T_{1 / 2}\right)$ half-life is for the decay of the parent nuclei ${ }^{302} \mathrm{Lv}$. The minimum value of logarithm $\left(T_{1 / 2}\right)$ half-life which is reference the behavior magic number $N=184$, this observation indicate the role of neutron shell in $\alpha$-decay.

From the Figure 1, it can be seen clearly the GLM, CPPM, Royer, UDL, VSS, SemFIS and DEKH are the same orientation. It should be taken into account that GLM values match close to Royer formula up to $\mathrm{N}=184$ rather than other model and formulae but after through this magic number the GLM is match close to SemFIS and Royer formulae.

The correlation between $Q$-value and logarithm $\left(\mathrm{T}_{1 / 2}\right) \alpha$ decay half-lives was shown in Figure 2, it show the logarithm $\left(\mathrm{T}_{1 / 2}\right) \alpha$-decay half-lives linearly decrease with increasing $Q$ value with 0.993 square correlation coefficient $\left(R^{2}\right)$.

\section{Conclusion}

The $\alpha$-decay half-lives for even-even $\mathrm{Lv}$ isotopes within GLM have been performed. The comparison between GLM, CPPM and some empirical formulae such as Royer, UDL, VSS, SemFIS and DEKH have been done, the result is match close to each other. From the result we predict by GLM, the minimum value of logarithm $\left(\mathrm{T}_{1 / 2}\right)$ half-life of even-even isotopes is founded in $N=184$, which is influence of the neutron magic number. Also we found the GLM is match close to Royer formula up to influence magic number, after this influence the GLM is also be so close to SemFIS formula.

\section{References}

[1] Gamow, G. (1928). Zur quantentheorie des atomkernes. Zeitschrift für Physik, 51 (3-4), 204-212.

[2] Zdeb, A., Warda, M., \& Pomorski, K. (2013). Half-lives for $\alpha$ and cluster radioactivity within a Gamow-like model. Physical Review C, 87 (2), 024308.

[3] Sandulescu, A., Poenaru, D. N., \& Greiner, W. (1980). New type of decay of heavy nuclei intermediate between fission and. cap alpha. decay. Sov. J. Particles Nucl.(Engl. Transl.);(United States), 11 (6).

[4] Warda, M., \& Robledo, L. M. (2011). Microscopic description of cluster radioactivity in actinide nuclei. Physical Review C, 84 (4), 044608.

[5] Royer, G. (2000). Alpha emission and spontaneous fission through quasi-molecular shapes. Journal of Physics G: Nuclear and Particle Physics, 26 (8), 1149.

[6] Royer, G., \& Gherghescu, R. A. (2002). On the formation and alpha decay of superheavy elements. Nuclear Physics A, 699 (3), 479-492.

[7] Ibrahim, T. T., \& Wyngaardt, S. M. (2014). Preformation factor and alpha decay half-lives of superheavy nuclei. Journal of Physics G: Nuclear and Particle Physics, 41 (5), 055111.

[8] Yao, Y. J., Zhang, G. L., Qu, W. W., \& Qian, J. Q. (2015). Comparative studies for different proximity potentials applied to $\alpha$ decay. The European Physical Journal A, 51 (9), 1-9.

[9] Sun, X. D., Guo, P., \& Li, X. H. (2016). Systematic study of $\alpha$ decay half-lives for even-even nuclei within a two-potential approach. Physical Review C, 93 (3), 034316.

[10] Javadimanesh, E., Hassanabadi, H., Rajabi, A. A., Rahimov, H., \& Zarrinkamar, S. (2012). Alpha Decay Half-Lives of Some Nuclei from Ground State to Ground State with Yukawa Proximity Potential. Communications in Theoretical Physics, $58(1), 146$.

[11] Santhosh, K. P., \& Priyanka, B. (2014). Heavy particle radioactivity from superheavy nuclei leading to ${ }^{298} 114$ daughter nuclei. Nuclear Physics A, 929, 20-37.

[12] Royer, G., \& Zhang, H. F. (2008). Recent $\alpha$ decay half-lives and analytic expression predictions including superheavy nuclei. Physical Review C, 77 (3), 037602.

[13] Qi, C., Xu, F. R., Liotta, R. J., \& Wyss, R. (2009). Universal decay law in charged-particle. 
[14] Dong, T., \& Ren, Z. (2005). New calculations of $\alpha$-decay halflives by the Viola-Seaborg formula. The European Physical Journal A-Hadrons and Nuclei, 26 (1), 69-72.

[15] Poenaru, D. N., Plonski, I. H., \& Greiner, W. (2006). $\alpha$-decay half-lives of superheavy nuclei. Physical Review C, 74 (1), 014312

[16] Denisov, V. Y., \& Khudenko, A. A. (2009). $\alpha$-decay half-lives: Empirical relations. Physical Review C, 79 (5), 054614.

[17] Krappe, H. J., \& Pomorski, K. (2012). Theory of Nuclear Fission: A Textbook (Vol. 838). Springer Science \& Business Media.

[18] Poenaru, D. N., Gherghescu, R. A., \& Greiner, W. (2011). Single universal curve for cluster radioactivities and $\alpha$ decay. Physical Review C, 83 (1), 014601.

[19] Blendowske, R., \& Walliser, H. (1988). Systematics of cluster-radioactivity-decay constants as suggested by microscopic calculations. Physical review letters, 61 (17), 1930.

[20] Hassanabadi, H., Javadimanesh, E., \& Zarrinkamar, S. (2013). A new barrier potential and alpha-decay half-lives of eveneven nuclei in the $82 \leq Z \leq 92$ regime. Nuclear Physics A, 906, 84-93.

[21] Royer, G., \& Moustabchir, R. (2001). Light nucleus emission within a generalized liquid-drop model and quasimolecular shapes. Nuclear Physics A, 683 (1), 182-206.

[22] Royer, G. (2010). Analytic expressions for alpha-decay halflives and potential barriers. Nuclear Physics A, 848 (3), 279291.

[23] Santhosh, K. P., \& Priyanka, B. (2014). Predictions for the $\alpha-$ decay chains of $Z=120$ superheavy nuclei in the range $272 \leq$ A $\leq 319$. Physical Review C, 90 (5), 054614.

[24] Qi, C., Xu, F. R., Liotta, R. J., Wyss, R., Zhang, M. Y.,
Asawatangtrakuldee, C., \& Hu, D. (2009). Microscopic mechanism of charged-particle radioactivity and generalization of the Geiger-Nuttall law. Physical Review C, $80(4), 044326$.

[25] Bao, X. J., Zhang, H. F., Dong, J. M., Li, J. Q., \& Zhang, H. F. (2014). Competition between $\alpha$ decay and cluster radioactivity for superheavy nuclei with a universal decay-law formula. Physical Review C, 89 (6), 067301.

[26] Santhosh, K. P., Sukumaran, I., \& Priyanka, B. (2015). Theoretical studies on the alpha decay of $178-220 \mathrm{~Pb}$ isotopes. Nuclear Physics A, 935, 28-42.

[27] Sobiczewski, A., Patyk, Z., \& Ćwiok, S. (1989). Deformed superheavy nuclei. Physics Letters B, 224 (1-2), 1-4.

[28] Santhosh, K. P., \& Priyanka, B. (2014). Predictions for the $\alpha$ decay chains of $Z=120$ superheavy nuclei in the range $272 \leq$ $\mathrm{A} \leq 319$. Physical Review C, 90 (5), 054614.

[29] Viola, V. E., \& Seaborg, G. T. (1966). Nuclear systematics of the heavy elements-II Lifetimes for alpha, beta and spontaneous fission decay. Journal of Inorganic and Nuclear Chemistry, 28 (3), 741-761.

[30] Akrawy, D. T., \& Poenaru, D. N. (2017). Alpha decay calculations with a new formula. arXiv preprint arXiv: 1702.05598 .

[31] Denisov, V. Y., \& Khudenko, A. A. (2010). Erratum: $\alpha$-decay half-lives: Empirical relations [Phys. Rev. C 79, 054614 (2009)]. Physical Review C, 82 (5), 059901.

[32] Denisov, V. Y., \& Khudenko, A. A. (2009). $\alpha$-Decay halflives, $\alpha$-capture, and $\alpha$-nucleus potential. Atomic Data and Nuclear Data Tables, 95 (6), 815-835.

[33] Denisov, V. Y., Davidovskaya, O. I., \& Sedykh, I. Y. (2015). Improved parametrization of the unified model for $\alpha$-decay and $\alpha$ capture. Physical Review C, 92 (1), 014602. 